\title{
Benefits of an 8-form Tai Chi Training Exercise on Balance Performance, Falling Risk, and Muscle Strengths in Elderly with Limited Strength: A Feasibility Study
}

\author{
Jarinee Jiraphan ${ }^{1 *}$, Praneet Pensri ${ }^{2}$, Pakpachong Vadhanasin ${ }^{3}$ and Chaipat Lawsirirat ${ }^{4}$ \\ ${ }^{1}$ Technopreneurship and Innovation Management Graduate School, \\ Chulalongkorn University, Bangkok, Thailand \\ ${ }^{2}$ Faculty of Allied Health Sciences, \\ Chulalongkorn University, Bangkok, Thailand \\ ${ }^{3}$ Faculty of Commerce and Accountancy, \\ ChulaloPak.v@acc.chula.ac.th \\ ${ }^{4}$ Faculty of Sports Science, \\ Chulalongkorn University, Bangkok, Thailand \\ ${ }^{*}$ Corresponding author's email: Jarinee.Jiraphan [AT] gmail.com
}

\begin{abstract}
The aim of this study was to determine the effectiveness of an 8-form Tai Chi training exercise on balance performance, falling risk, and muscle strengths in elderly with limited strength. Twenty-six females between 70-85 years old with balance problem and moderate lower extremity muscular strengths (grade 2+ to 3+) without prior Tai Chi training volunteered for the study. The participants underwent the 8-form Tai Chi training exercise for 45 minutes/session, 3 sessions a week for 12 weeks. Balance performance (m-CTSIB, Postural Stability Balance Test, and 8-foot up-and-go Test), falling risk (Fall Risk Overall Stability Test and Fear of Falling Assessment), and muscle strengths (30-second Chair Stand Test and Pressure Biofeedback Unit Test) were assessed during pretest, mid-test, and post-test. One-way repeated measures ANOVA was conducted at the significant level of 0.05. The results after 6 weeks of training showed that balance and falling risk were improved where 8 foot up-and-go test and fear of falling assessment were significantly better than pre-experiment, while after 12 weeks, balance, falling risk, and lower extremity muscular strengths were significantly improved when compared to the pre-experiment. The 12-week 8-form Tai Chi training exercise was safe and effective in improving balance, falling risk, and lower extremity muscular strengths in ageing people with limited strengths.
\end{abstract}

Keywords - Tai Chi, balance, ageing, fear of falling, muscle strength

\section{INTRODUCTION}

Balance problem is a common cause of falling injury in senior citizen. Fall-induced injuries are a leading cause of disability and become a great threat to health care system and senior housing $[1,2]$. Training exercise which helps improve balance is, thus, necessary for frequented fallen people or people with mobility problems and senior citizen. Several types of exercise trainings, such as, walking, Tai Chi, or yoga, have been recommended to elderly with balance problem.

Tai Chi is very popular among elderly. It requires them to be mindful of their movements through breathing technique while helps improve lower muscular strengths [3]. Generally, Tai Chi consists of 20-100 movements taking more than 20 minutes duration/session [4-6]. Depending on objective of exercise, Tai Chi has been modified to various forms and were extensively studied [7-11]. However, the benefit of Tai Chi exercise on balance and related issues such as falling risk is far from conclusive where some studies showed no improvement on balance after Tai Chi exercise [12-14].

The purpose of this study was to assess the effect of a 12-week 8-form Tai Chi program on balance performance, falling risk, and muscle strengths. While there were several studies using Tai Chi in elderly, this study offered a unique contribution by first focusing on elderly who were between 70-85 years of age with limited muscle strengths. This group 
of population were growing in number and needed attentions in order to prevent them from becoming a bedridden. The population in our study were, therefore, much older and relatively frailer than population studied in literature. The second contribution was that the study focused on comprehensive assessment of balance where both static and dynamic balance were tested. Lastly, the study was applied in a senior housing with limited human and financial resources. With several constraints, the study was designed as a feasibility study with one group. The study was hypothesized that after 12 weeks of training, a 12-week 8-form Tai Chi training exercise improved balance performance, falling risk, and muscle strengths in elderly with limited strengths.

\section{METHODS}

\subsection{Participants}

Twenty-nine sedentary female elderly, who lived in an elderly female foster home at Pathum Thani province, Thailand, participated in this study. All twenty-nine participants fulfilled the following inclusion criteria: 1) Age between 70-85 years 2) No serious disease that doctors did not allow to exercise 3) Never had any experience in practicing Tai Chi 4) Strengths of lower extremity muscles were moderate (grade 2+ to 3+) after assessment by a certified physical therapist using Manual Muscle Testing (MMT) [15] and 5) at least one of four test results were lower than normative values. The four tests were 8-foot up-and-go Test, Overall m-CTSIB Test, Overall Postural Stability Test, and Fall Risk Test. Overall m-CTSIB Test, Overall Postural Stability Test, and Fall Risk Test were assessed using BIODEX Balance System SD (Model \#950-441, New York, USA). The cutoff points for these three tests were set using normative value reported by the manufacture, while the criteria for 8 -foot up-and-go test was adopted from the work by Jones and Rikli [16]. The average age, height, and weight of the participants were $76 \pm 5.41$ years, $148 \pm 5.61 \mathrm{~cm}$, and 59 $\pm 12.20 \mathrm{~kg}$, respectively. Though we did not include the history of falling in the inclusion criteria, our interview with the participants showed that every participant who passed the inclusion criteria fell at least once over 1 year ago, and $66 \%$ experienced at least 1 fall in the past 6 months.

\subsection{Intervention}

A modified Tai Chi training exercise was created from a careful selection of Tai Chi forms from a 24-form Tai Chi training program [17]. Prior to the study, a focus group with elderly was conducted. From the focus group, it was commented that the 24-form Tai Chi was difficult to remember, follow and execute. A more concise but effective Tai Chi exercise was, thus, required especially for our target participants. To create an effective Tai Chi exercise, Tai Chi forms were carefully chosen such that they required lower extremity muscle works and movements in three planes, and disturbed body balance. These criteria allowed the participants to train on developing balance as well as improving lower body strength. Following this guideline, 12 Tai Chi forms were selected. A pilot study of EMG analysis with an elderly was, then, conducted. EMG was attached to the following muscles, i.e., 1) Gluteus Medius, 2) Vastus Medialis, 3) Rectus Femoris, 4) Bicep Femoris, 5) Tibialis Anteria and 6) Gastrocnemius. To minimize Tai Chi forms while maintain its effectiveness, Tai Chi forms were adopted if the root mean square of EMGs of at least three muscles were greater than 100 micro volts. Finally, an 8-form of Tai Chi (as shown in Figure 1) was created as the training exercise for 12 weeks.

The participants were trained 3 sessions a week. Each session took 45 minutes with 10 minutes warm up and 5 minutes cool down. The protocol for the study was presented in Figure 2. The training was progressive every 4 weeks. The participants were asked to perform only forms 1-6 in the first 4 weeks, forms 1-7 in the second 4 week, and forms 1 8 in the last 4 weeks. Progressive training allowed the participants to familiarize with the training exercise and increase challenges to improve muscle strengths.

All participants understood the risks involved and signed a consent form before joining the program. The experiment protocol was approved by the ethical review committee for research involving human research subjects of Chulalongkorn University. To ensure maximum safety, the participants were asked to hold the chair or other support facilities throughout their exercise during beginning of training sessions [18]. They were encouraged to let go off the chair if they felt more confidence. One nurse and 4 nursing aids were stationed in every training session to ensure maximum safety and correct Tai Chi postures. The participants were requested not to have any other physical activities during our 12 week Tai Chi training exercise. 


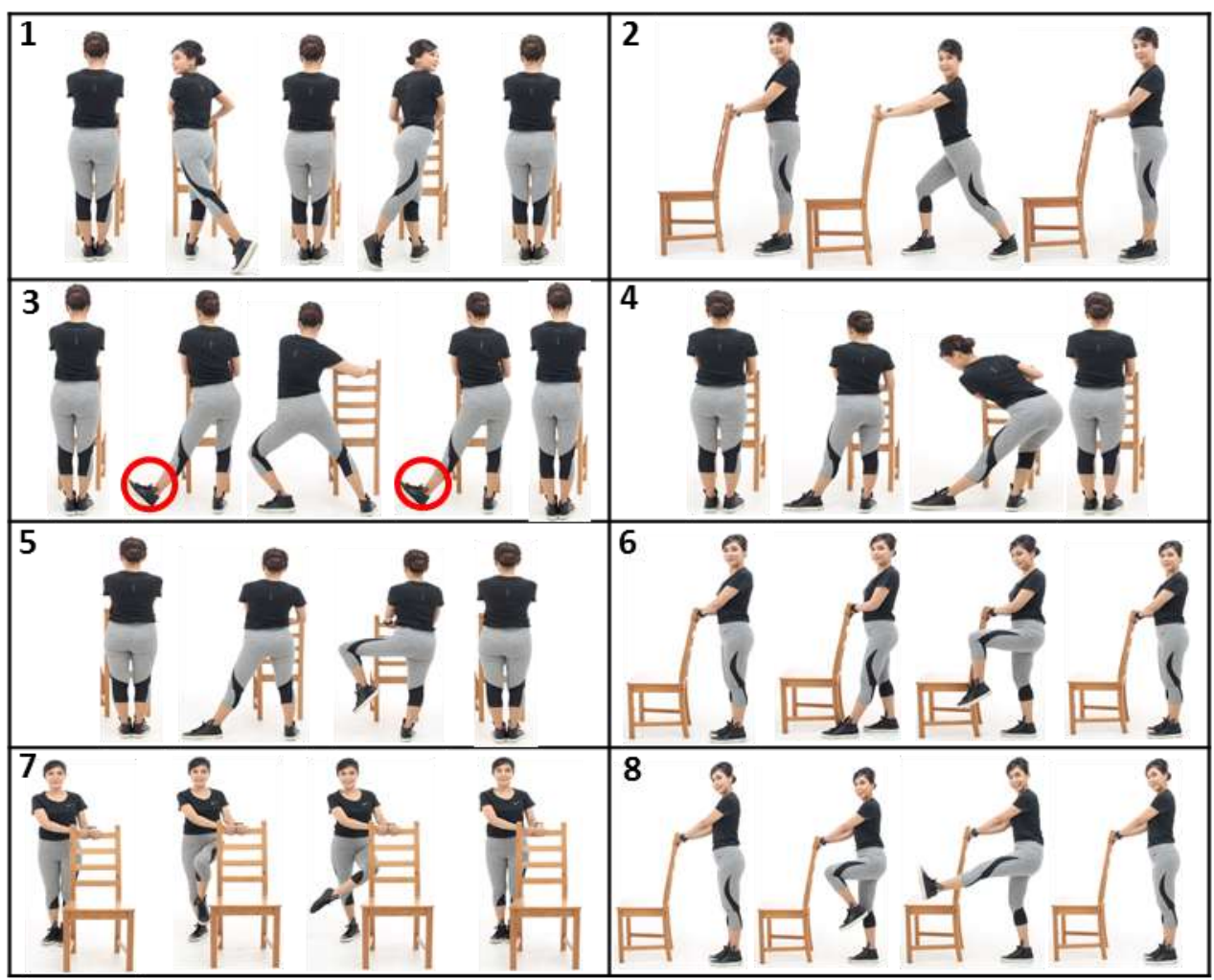

Figure 1: An 8-Form Tai Chi Training Exercise

Week 1-4
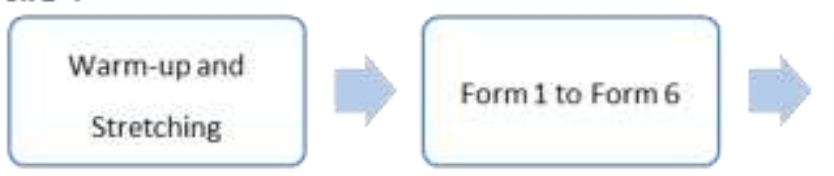

Cool-down Exercise

Week 5-8

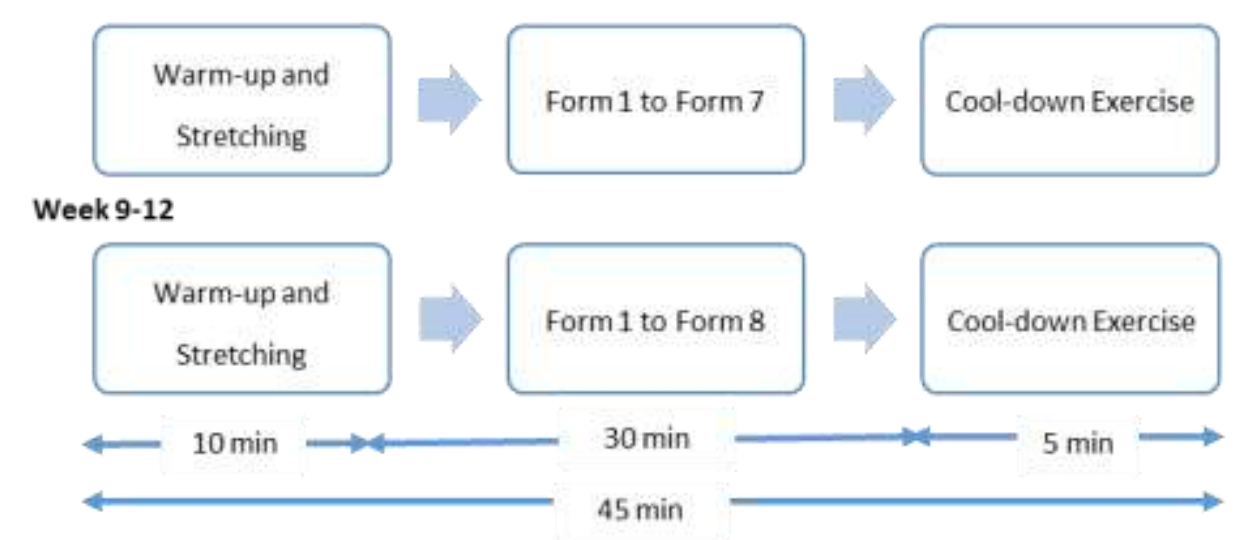

Figure 2: Protocol of an 8-form Tai Chi Training Exercise

\subsection{Outcome measurements and Data Collections}

This research studied the effectiveness of an 8-form Tai Chi training exercise in elderly on balance performance, falling risk, and muscle strengths. The balance test included both static and dynamic balance tests. The static balance test was assessed using BIODEX Balance System SD (Model \#950-441, New York, USA). For static balance test, the participants were required to perform modified clinical test for sensory integration and balance test (m-CTSIB) and Postural Stability Balance Test (PSBT). 8-foot up-and-go test (TUG) was used to assess dynamic balance. Falling risk was assessed using Fall Risk Overall Stability Test (FROST) using BIODEX Balance System SD and a 34-question fear of falling assessment questionnaire (FFA). 30-second chair stand test (CST) and Pressure Biofeedback Unit Test (PBUT) were employed to assess lower extremity and core muscle strengths. m-CTSIB, PSBT, and FROST measured stability 
index reported by BIODEX Balance System SD. The output measurements were collected three times at pretest (3 days before the intervention), mid-test (3 days after 6 weeks), and post-test (3 days after 12 weeks).

\subsubsection{Balance Performance}

Static Balance: m-CTSIB and PSBT were utilized to assess static balance. Two subtests of m-CTSIB from four subtests (eye-opened/eye-closed on firm/foam surface) were used to prevent exhaustion. The two tests were eye-opened on firm surface and eye-closed on foam surface because they were the easiest and most challenging tasks in m-CTSIB. In $\mathrm{m}$-CTSIB, the participants were asked to stand on firm/foam surface with eye-opened/eye-closed for 30 seconds while the machine measured stability index and sway index. The participants performed two sets with 10 seconds rest. PSBT emphasized on the ability to maintain center of balance. The participants were asked to move cursor appeared on screen to the center of the monitor for 20 seconds in 3 sets with a 10-sec rest. Sway index in anterior/posterior and medial/lateral axis and overall stability index were reported.

Dynamic Balance: 8-foot up-and-go test (TUG) was employed. The participants performed TUG three times with 30second rest interval between tests. The maximum value was reported.

\subsubsection{Falling Risk}

The participants were assessed falling risk using FROST from BIODEX Balance System SD and a 34-question fear of falling assessment questionnaire. FROST aimed to identify potential fall candidates. To assess FROST, the participants were asked to stand on firm surface with eye-opened/eye-closed in normal foot standing away or $7.5 \mathrm{~cm}$ foot standing away. The participants performed FROST three times and the average value was reported.

Fear of Falling Assessment (FFA) was assessed using a questionnaire to measure fear of falling in Thai elderly. The questionnaire had 34 questions, consisting of 3 main factors, 15 questions in physical fitness factor, 8 questions in environmental factor, and 11 questions in psychosocial factor. The participants responded to questions read by the researcher. Participant with fear of falling will receive a test score of 66 or more [19].

\subsubsection{Muscle Strengths}

Lower extremity muscles: 30 -second Chair Stand Test was used to evaluate lower extremity muscles. The participants performed CST three times with a 30-second interval rest. The maximum value was reported.

Core muscles: Core muscles were tested due to their importance in postural stability control. PBUT was utilized to assess core muscle strengths. The participants were asked to lie down in prone position with arms to each side. The pressure biofeedback unit (Stabilizer Pressure Biofeedback Unit, Chattanooga Group, Inc. USA) was placed under the lower abdomen, and the participants were asked to contract their abdomen following instructions. [20] PBUT was tested three times and the maximum value was reported.

The tests were divided into 5 stations. The first station was BIODEX Balance System Test. The participants were assessed static balance using m-CTSIB and PSBT and falling risk using FROST. This station took around 10 minutes. The second station was Pressure Biofeedback Unit Test which took around 5 minutes. The participants performed 8 footup-and-go test in station 3 for 5 minutes before responding to the fear of falling assessment questionnaire in station 4 for 3 minutes. Finally, 30-second chair stand was conducted in station 5 for 3 minutes. The rest interval between stations was 3 minutes, and the overall time spent during the test was 38 minutes.

\subsection{Qualitative Assessment}

During intervention, the participants were encouraged to give feedbacks and discuss with the other participants on the intervention in order to promote attendance and social gathering among the participants.

\subsection{Statistical Analysis}

Statistical analyses were performed using SPSS 22.0 (SPSS Inc., IL, USA). Mean and standard deviation were reported. One-way repeated measures ANOVA was conducted to test the differences between pretest, mid-test and posttest of balance performance (m-CTSIB, PSBT, and TUG), falling risk (FROST and FFA), and muscle strengths (CST and PBUT). The level of significance was set at 0.05. A pair-wise analysis was conducted using Bonferroni method.

\section{RESULTS}

From the 29 participants who passed the prescreening and joined the program, 3 participants dropped out due to health problem. The average number of attendances was 32 days from 36 days (89\%).

The means and standard deviations of balance performance, falling risk, and muscle strengths assessed at pretest (Week 0), mid-test (Week 6), and post-test (Week 12) were reported in Table 1. 
Table 1: One-way repeated measures ANOVA analysis

\begin{tabular}{|c|c|c|c|c|c|c|c|c|}
\hline \multirow{2}{*}{\multicolumn{3}{|c|}{ Output measurements }} & & \multicolumn{3}{|c|}{ Time of Assessment } & \multirow{2}{*}{ p-value } & \multirow{2}{*}{$\mathbf{E S}$} \\
\hline & & & & Week 0 & Week 6 & Week 12 & & \\
\hline \multicolumn{9}{|c|}{ Static Balance Test } \\
\hline \multirow{15}{*}{ 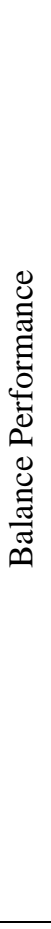 } & \multirow{6}{*}{ 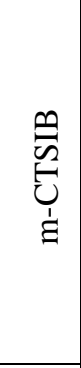 } & \multirow{2}{*}{ Eye-Opened Firm Surface ${ }^{a}$} & Mean & 1.76 & 1.31 & 1.34 & \multirow{2}{*}{$0.034 *$} & \multirow{2}{*}{0.147} \\
\hline & & & SD & 1.07 & 0.33 & 0.54 & & \\
\hline & & \multirow{2}{*}{ Eye-Closed Foam Surface } & Mean & 5.46 & 5.21 & 5.55 & \multirow{2}{*}{0.413} & \multirow{2}{*}{0.035} \\
\hline & & & SD & 1.10 & 1.43 & 1.55 & & \\
\hline & & \multirow{2}{*}{ Overall Stability } & Mean & 3.60 & 3.25 & 3.44 & \multirow{2}{*}{0.059} & \multirow{2}{*}{0.107} \\
\hline & & & SD & 0.78 & 0.81 & 0.89 & & \\
\hline & \multirow{6}{*}{ 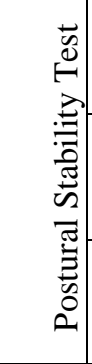 } & \multirow{2}{*}{ Anterior/Posterior ${ }^{\mathrm{a}}$} & Mean & 2.58 & 1.77 & 1.49 & \multirow{2}{*}{$0.025^{*}$} & \multirow{2}{*}{0.156} \\
\hline & & & SD & 2.24 & 1.20 & 1.13 & & \\
\hline & & \multirow{2}{*}{ Medial/Lateral } & mean & 2.83 & 1.97 & 1.82 & \multirow{2}{*}{$0.040 *$} & \multirow{2}{*}{0.121} \\
\hline & & & SD & 2.49 & 1.41 & 1.16 & & \\
\hline & & \multirow{2}{*}{ Overall Stability $^{\mathrm{a}}$} & mean & 4.34 & 2.92 & 2.60 & \multirow{2}{*}{$0.003 *$} & \multirow{2}{*}{0.226} \\
\hline & & & SD & 2.83 & 1.60 & 1.41 & & \\
\hline & \multicolumn{8}{|c|}{ Dynamic Balance Test } \\
\hline & \multirow{2}{*}{\multicolumn{2}{|c|}{8 -foot up-and-go test ${ }^{\mathrm{a}}(\mathrm{sec})$}} & mean & 12.67 & 9.81 & 9.97 & \multirow{2}{*}{$0.000 *$} & \multirow{2}{*}{0.546} \\
\hline & & & SD & 4.70 & 3.51 & 3.46 & & \\
\hline \multirow{4}{*}{ 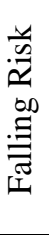 } & Foll $\mathrm{t}$ - a & ick Overoll Stahilitya & mean & 2.73 & 2.40 & 2.08 & $0047 *$ & 0132 \\
\hline & & & SD & 1.42 & 0.55 & 0.67 & & \\
\hline & Fear & f Falling Assessment ${ }^{\mathrm{a}}$ & mean & 66.65 & 65.08 & 61.85 & $0000 *$ & 0384 \\
\hline & (test & cores) & SD & 19.47 & 17.29 & 14.70 & & \\
\hline & & & Lower & xtremity $\mathrm{N}$ & uscles & & & \\
\hline 点 & 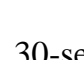 & cond Chair Stand Test (counts) & mean & 12.15 & 13.08 & 15.27 & $0000 *$ & 0388 \\
\hline 离 & & & SD & 3.03 & 3.44 & 2.49 & & \\
\hline$\frac{0}{0}$ & & & & re Muscle & & & & \\
\hline 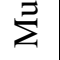 & Press & ure Biofeedback Unit Test & mean & 2.23 & 3.23 & 4.77 & $0000 *$ & 0316 \\
\hline & $(\mathrm{mm}$ & & SD & 2.07 & 2.72 & 2.47 & & \\
\hline
\end{tabular}

ap-value was adjusted by Greenhouse-Geiser Method.

Table 1 presented one-way repeated measures ANOVA result and reported p-values and effect sizes of each outcome variable. Green-Geisser Method was utilized to adjust for $\mathrm{p}$-value should the assumption of sphericity violated. The results showed that every outcome measure except stability index of Eye-Closed Foam Surface test and Overall Stability Index of m-CTSIB was statistically significant at 0.05 level. As seen from Table 1, the results showed medium to large effect size where ES was greater than 0.06 and 0.14. Pair-wise analysis conducted using Bonferroni method was reported in Table 2. Four outcome measures, i.e., Eye-Opened Firm Surface Stability Index, Overall Postural Stability Index, time of 8-foot up-and-go test, and fear of falling assessment test score showed significant improvement at mid-test when compared to pretest, while all outcome measures excepts m-CTSIB showed significant improvement at post-test when compared to pretest. 
Table 2: Post hoc analysis

\begin{tabular}{|c|c|c|c|c|c|}
\hline & \multirow{2}{*}{\multicolumn{2}{|c|}{ Output measurements }} & \multicolumn{3}{|c|}{ Time of Assessment } \\
\hline & & & Week 0 & Week 6 & Week 12 \\
\hline \multirow{9}{*}{ 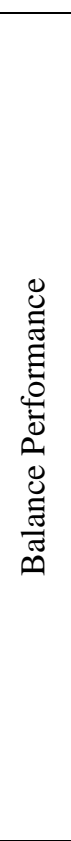 } & \multicolumn{5}{|c|}{ Static Balance Test } \\
\hline & \multirow{3}{*}{ 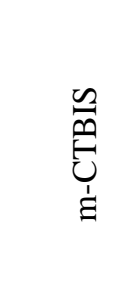 } & Eye-Opened Firm Surface ${ }^{\mathrm{a}}$ & Week 6 & Week 0 & \\
\hline & & Eye-Closed Foam Surface & & & \\
\hline & & Overall Stability & & & \\
\hline & \multirow{3}{*}{ 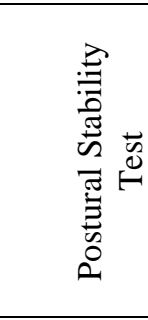 } & Anterior/Posterior & Week 12 & & Week 0 \\
\hline & & Medial/Lateral $^{\mathrm{a}}$ & Week 12 & & Week 0 \\
\hline & & Overall Stability & $\begin{array}{c}\text { Week } 6 \\
\text { Week } 12 \\
\end{array}$ & Week 0 & Week 0 \\
\hline & \multicolumn{5}{|c|}{ Dynamic Balance Test } \\
\hline & 8 -foot up- & and-go test & $\begin{array}{c}\text { Week } 6 \\
\text { Week } 12\end{array}$ & Week 0 & Week 0 \\
\hline \multirow{2}{*}{ 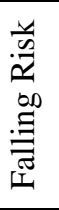 } & \multicolumn{2}{|c|}{ Fall Risk Overall Stability } & Week 12 & & Week 0 \\
\hline & \multicolumn{2}{|c|}{ Fear of Falling Assessment } & $\begin{array}{c}\text { Week } 6 \\
\text { Week } 12\end{array}$ & $\begin{array}{c}\text { Week } 0 \\
\text { Week } 12\end{array}$ & $\begin{array}{l}\text { Week } 0 \\
\text { Week } 6\end{array}$ \\
\hline \multirow{4}{*}{ 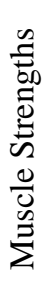 } & \multicolumn{5}{|c|}{ Lower Extremity Muscles } \\
\hline & \multicolumn{2}{|c|}{ 30-second Chair Stand Test } & Week 12 & Week 12 & $\begin{array}{l}\text { Week } 0 \\
\text { Week } 6\end{array}$ \\
\hline & \multicolumn{5}{|c|}{ Core Muscles } \\
\hline & \multicolumn{2}{|c|}{ Pressure Biofeedback Unit Test } & Week 12 & Week 12 & $\begin{array}{l}\text { Week } 0 \\
\text { Week } 6\end{array}$ \\
\hline
\end{tabular}

apair-wise comparison was conducted using LSD after Bonferroni found no significant pair comparison.

All participants (26/26 participants) reflected that they felt improvement in muscle strengths and had more confident when walking and no longer fear of falling. After the training intervention, 20 participants continued practicing the 8 form Tai Chi training exercise and formed up a group as a routine gathering in the senior housing. The participants reported the benefits of exercise and relationship in the exercise group. Moreover, they also reported that they got deep sleep more often. Another benefit of the training program reported by the participants was the selected forms of the 8form Tai Chi were easy to memorize and follow enabling the participants to lead the group and train newcomers.

\section{DISCUSSION}

In our study, a 12-week training of an 8-form of Tai Chi exercises improved balance performance, falling risk, and muscle strengths. The results agreed with several other studies applying Tai Chi in elderly where Tai Chi training improved on balance, risk of falling, and physical ability [2, 10, 21-26]. Tai Chi was able to improve proprioception of elderly and, thus, helped improve balance and falling risk [27]. However, our result disagreed with the results from Hägglund, Boman, and Brännström [28] where they found no benefit of Tai Chi training in 70-year old elderly with chronic heart failure. The difference was possibly due to our exercise intensity and progressive training allowing frailer elderly to see improvement after training.

The improvements were noticeable as early as 6 weeks after Tai Chi training which was consistent with Au-Yeung, Hui-Chan, Tang [29] and Tsang, Hui-Chan [30] where Tai Chi could improve balance in four weeks. Early positive results of Tai Chi training in our study was because our careful selection strategy of Tai Chi forms from the pilot study of EMG emphasized on the works of lower extremity muscles. Appropriate exercise with enough intensity improved strength and balance in sedentary elderly and community dwelling [31-32]. One noticeable improvement after 6 weeks of 
training was Fear of Falling Assessment which was self-assessed using a questionnaire. The results indicated that after training the participants had more confidence and belief that they were stronger even if the data did not suggest that the participants were significantly stronger than pretest. Since the participants had experienced at least one fall over the past year, the results proved that the 8-form Tai Chi training exercise was effective in reducing fear of falling.

Our findings demonstrated the 8-form Tai Chi training exercise for 12 weeks benefited relatively frail elderly in senior housing by improving balance performance, falling risk, and muscle strengths. While researches always reported the intensity of Tai Chi training, only few reported the number of forms of Tai Chi and detailed instructions [6]. The feedbacks from our participants suggested the total number of forms of Tai Chi was important as it encouraged the participants to attend training sessions especially in relatively frail and older elderly. Too complicated, complex, and many forms of Tai Chi discouraged the participants to continue Tai Chi training. In this study, the participants' attendance was $89 \%$ indicating that the session was fun and easy to follow. In addition, since our focus was on more than 70 years old participants, we needed to be extra careful of selected forms of Tai Chi. In literature, 10-12 forms of Tai Chi were often used as an intervention [29, 33-35]. Only a few researches adopted 8-form Tai Chi and found improvement after training [2]. Our finding emphasized that there was no need to include many forms of Tai Chi for exercise in order to be effective. Only 8 forms of Tai Chi were sufficient enough to improve balance performance, falling risk, and muscle strengths.

Our study also supported the application of Tai Chi in senior housing environment. As resources in subsidized senior housing were limited and the number of elderly increased drastically in senior housing, Tai Chi was an effective training exercise that could improve quality of life, fall prevention, functional fitness as well as reduce cost of health care in senior housing $[3,36]$.

The drawback of this study needs to be addressed. The effect of the training cannot be determined without a control group. A large-scale clinical trial should be undertaken to determine if the 8-form Tai Chi training exercise affects balance performance, falling risk, and muscle strengths. Despite these limitations, our preliminary findings demonstrate that the 8-form Tai Chi training exercise is an enjoyable, safe, and potentially effective approach to improve physical activity in senior housing. The major strength and contribution of our study lie in the simplicity of Tai Chi forms which are easy to follow and remember yet effective in improving balance and strengths in relatively frail elderly.

\section{CONCLUSIONS}

Falls are common threat in elderly. Poor balance and muscle strengths are leading factors to falls. This research provided an effective 8-form Tai Chi training exercise for limited strength elderly in senior housing. The study showed that balance, falling risk, and muscle strengths were able to improve after Tai Chi training for 45 minutes per session, 3 sessions a week for 12 weeks. Many variables showed improvement as early as 6 weeks of training. In conclusion, the proposed 8-form Tai Chi has potential to be adopted among elderly people with limited strength and is very effective when applied in senior housing.

\section{REFERENCES}

[1] Liu JYW, Kwan RYC, Lai CK, Hill KD, “A simplified 10-step Tai-chi programme to enable people with dementia to improve their motor performance: a feasibility study", Clin Rehabil, vol. 32, no. 12, pp.:1609-1623, 2018.

[2] Jie Z, Huang L, Wu Y, Zhang Y, "The effectiveness of a combined exercise intervention on physical fitness factors related to falls in community-dwelling older adults" Clin Interv Aging, vol. 9, pp. 131-40, 2014.

[3] Brad M, Matt L, Margaret MG, Adrienne C, Peter MW, Lewis AL, "Functional benefits of tai chi training in senior housing facilities", J Am Geriatr Soc, vol. 62, No.8, pp. :1484-1489, 2014.

[4] Del PC, E Obrero G, Lomas V, "The Effect of Tai Chi on Reducing the Risk of Falling: A Systematic Review and Meta-Analysis", Am J Chin Med, vol. 44, no. 5, pp. 895-906, 2016.

[5] Du S, Dong J, Zhang H, Jin S, Xu G, Liu Z, et al, "Taichi exercise for self-rated sleep quality in older people: A systematic review and meta-analysis", Int J Nurs Stud, vol. 52 no. 1, pp. 368-79, 2015.

[6] Wu Y, MacDonald HV, Pescatello LS, "Evaluating Exercise Prescription and Instructional Methods Used in Tai Chi Studies Aimed at Improving Balance in Older Adults: A Systematic Review”, J Am Geriatr Soc, vol. 64, no. 10, pp. 2074-2080, 2016.

[7] Wolf SL, Coogler C, Xu T, "Exploring the basis for Tai Chi Chuan as a therapeutic exercise approach", Arch Phys Med Rehabil, vol. 78 no. 8, pp. 886-892, 1997.

[8] Wolf SL, Sattin RW, Kutner M, O'Grady M, Greenspan AI, Gregor RJ, "Intense Tai Chi Exercise Training and Fall Occurrences in Older, Transitionally Frail Adults: A Randomized, Controlled Trial”, J Am Geriatr Soc, vol. 51, no. 12, pp. 1693-1701, 2003.

[9] Yu DH, Yang HX, "The effect of Tai Chi intervention on balance in older males", Journal of sport and Health science, vol. 1, no. 1, pp. 57-60, 2012.

[10] Ni M, Mooney K, Richards L, Balachandran A, Sun M, Harriell K, et al., "Comparative impacts of Tai Chi, balance training, and a specially-designed yoga program on balance in older fallers”, Arch Phys Med Rehabil, vol. 95, no. 9, pp. 1620-1628.e30, 2014. 
[11] Lelard T, Ahmaidi S, "Effects of physical training on age-related balance and postural control", Neurophysiol Clin, vol. 45, no. 4-5, pp. 357-369, 2015.

[12] Logghe I, Zeeuwe P, Verhagen A, Wijnen-Sponselee R, Rademaker A, Willemsen S, et al., Tai Chi Chuan does not reduce the risk of falls in older people. Huisarts en Wetenschap, vol. 52, no. 11, pp. 536-541, 2009.

[13] Logghe IHJ, Verhagen AP, Rademaker ACHJ, Bierma-Zeinstra SMA, Rossum E van, Faber MJ, et al., "The effects of Tai Chi on fall prevention, fear of falling and balance in older people: A meta-analysis", Prev Med, vol. 51, no. 3-4, pp. 222-227, 2010.

[14] Logghe IHJ, Zeeuwe PEM, Verhagen AP, Wijnen-Sponselee RMT, Willemsen SP, Bierma-Zeinstra SMA, et al., "Lack of effect of tai chi chuan in preventing falls in elderly people living at home: A randomized clinical trial", J Am Geriatr Soc, vol. 57, no. 1, pp. 70-75, 2009.

[15] Clarkson HM, Musculoskeletal assessment: joint motion and muscle testing, Wolters Kluwer/Lippincott Williams \& Wilkins Health, USA, 2013.

[16] Jones CJ, Rikli RE, "Measuring functional”, The Journal on active aging, vol. 1, no. 24-30, 2002

[17] Loretta MW, Anatomy of Fitness: Tai Chi, Hinkler Books Pty Ltd., Australia, 2013.

[18] Kevin A, Katie R, Philippa L, P, Adam LG, Rowan HH, Tahir M. T. Chair-based exercises for frail older people: a systematic review. Biomed Res Int, vol. 2013, article ID 309506, 2013

[19] Punpisa S, Mantana V, Vimonwan H, Jaranit K, "Development of a Geriatric Fear of Falling Questionnaire for Assessing the Fear of Falling of Thai Elders", Journal of Physical Therapy Science, vol. 24, no. 4, pp. 359-364, 2012.

[20] Katharina von G, Kristin K, Berid R B, Ulrike K, Sabine W, Thomas E, et al., "Reliability of a test measuring transversus abdominis muscle recruitment with a pressure biofeedback unit", Physiotherapy, vol. 95, no. 1, pp. 8$14,2009$.

[21] Jia X, Jiang C, Tao J, Li Y, Zhou Y, Chen LD, "Effects of core strength training combined with Tai Chi Chuan for the musculoskeletal system and cardiopulmonary function in older adults: A study protocol for a randomized controlled trial", Medicine, vol. 97, no. 35, e12024, 2018.

[22] Huang CW, Chen WH, Chu HH, Jiang BC, Abbod M, Shieh JS, "Simple tai chi exercise for improving elderly postural stability via complexity index analysis", Artificial Life and Robotics, vol. 20, no. 1, pp. 42-48, 2015.

[23] Yildirim P, Ofluoglu D, Aydogan S, Akyuz G, "Tai Chi vs. combined exercise prescription: A comparison of their effects on factors related to falls", J Back Musculoskelet Rehabil, vol. 29, no. 3, pp. 493-501, 2016.

[24] Son NK, Ryu YU, Jeong HW, Jang YH, Kim HD, "Comparison of 2 Different Exercise Approaches: Tai Chi versus Otago, in Community-Dwelling Older Women”, J Geriatr Phys Ther, vol. 39, no. 2, pp. 51-57, 2016.

[25] Alsubiheen A, Petrofsky J, Daher N, Lohman E, Balbas E, "Effect of Tai Chi exercise combined with mental imagery theory in improving balance in a diabetic and elderly population", Med Sci Monit, vol. 21, pp. 3054-3061, 2015.

[26] Li L, Manor B, "Long term Tai Chi exercise improves physical performance among people with peripheral neuropathy. The American journal of Chinese medicine”, vol. 38, no. 03, pp. 449-459, 2010.

[27] Guo LY, Yang CP, You YL, Chen SK, Yang CH, Hou YY, et al., "Underlying mechanisms of Tai-Chi-Chuan training for improving balance ability in the elders", Chin J Integr Med, vol. 20, no. 6, pp. 409-415, 2014.

[28] Hägglund L, Boman K, Brännström M, “A mixed methods study of Tai Chi exercise for patients with chronic heart failure aged 70 years and older”, Nursing Open, vol. 5, no. 2, pp. 176-185, 2018.

[29] Au-Yeung SSY, Hui-Chan CWY, Tang JCS, "Short-form Tai Chi Improves Standing Balance of People with Chronic Stroke", Neurorehabil Neural Repair, vol. 23, no. 5, pp. 515-522, 2009.

[30] Tsang WW, Hui-Chan CW, "Effect of 4- and 8-wk intensive Tai Chi Training on balance control in the elderly", Med Sci Sports Exerc, vol. 36, no. 4, pp. 648-657, 2004.

[31] Pinto RS, Correa CS, Radaelli R, Cadore EL, Brown LE, Bottaro M, "Short-term strength training improves muscle quality and functional capacity of elderly women”, Age, vol. 36, no. 1, pp. 365-372, 2014.

[32] Timmons JF, Minnock D, Hone M, Cogan KE, Murphy JC, Egan B, "Comparison of time-matched aerobic, resistance, or concurrent exercise training in older adults”, Scand J Med Sci Sports, vol. 28, no. 11, pp. 2272-2283, 2018.

[33] Zhang JG, Ishikawa-Takata K, Yamazaki H, Morita T, Ohta T, "The effects of Tai Chi Chuan on physiological function and fear of falling in the less robust elderly: An intervention study for preventing falls", Arch Gerontol Geriatr, vol. 42, no. 2, pp. 107-116, 2006.

[34] Liu YWJ, Tsui CM, "A randomized trial comparing Tai Chi with and without cognitive-behavioral intervention (CBI) to reduce fear of falling in community-dwelling elderly people", Arch Gerontol Geriatr, vol. 59, no. 2, pp. 317-325, 2013.

[35] Liu H, A Frank, "Tai chi as a balance improvement exercise for older adults: A systematic review”, J Geriatr Phys Ther, vol. 33, no. 3, pp. 103-109, 2010.

[36] Wayne PM, Gagnon MM, Macklin EA, Travison TG, Manor B, Lachman M, et al., "The Mind Body-Wellness in Supportive Housing (Mi-WiSH) study: Design and rationale of a cluster randomized controlled trial of Tai Chi in senior housing”, Contemp Clin Trials, vol. 60, no. 96-104, 2017. 